\title{
26406 - REACTIVE OXYGEN SPECIES (ROS) SCAVENGER EXHIBITS ANTINOCICEPTIVE PROPERTIES IN THE RAT (SPRAGUE-DAWLEY) FORMALIN TEST.
}

\section{Won Hyung Lee Medical doctor, professor, Young Ho Lee, Medical Doctor, prefessor; Chungnam National Univrsity Hospital, Taejeon, , South Korea}

Introduction: ROS have been implicated in many degenerative neurological conditions. Recently, ROS is suggested to play an important role in pain modulation. This study is to assess the systemic and intrathecal effects of ROS scavenger, phenyl-N-tert-butylnitrone $(\mathrm{PBN})$, in rats subjected to the formalin test.

Methods: The experimental procedures were performed in accordance with the animal care guideline of NIH and the Korean Academy of Medical Science. 5\% formalin was injected in the left hind paw of Sprague-Dawley rats (N=70, 200-250g) after injection of various dose of intraperitoneal (IP group) PBN (saline, 20mg/kg, $50 \mathrm{mg} / \mathrm{kg}, 100 \mathrm{mg} / \mathrm{kg}$ ), or intrathecal (IT group) PBN (saline, $0.3 \mathrm{mg}, 1 \mathrm{mg}$ ) through the implanted lumbar spinal catheter. Number of flinches was measured for $60 \mathrm{~min}$ after formalin injection.

To evaluate the nitration of protein - nitrotyrosine (the marker of oxidative stress) - in lumbar spinal cord, the immunohistochemistry with antinitrotyrosine antibodies was performed in all groups after formalin injection and in normal(naive) rat.

Results: Formalin injected into the rat hind paw induced a biphasic nociceptive behaviour. IP group, nor IT group diminished the nociceptive behaviors dosedependently during the phase $¥$ and $¥ \pm$.

The immunohistochemical staining of nitrated proteins was nearly undetectable in normal(naive) rats. The immunohistochemical evaluation in saline pre-teated rats of both groups revealed the increase of nitrated proteins in gray matter in the lumbar spinal cord, and significantly diminished upon IP or IT PBN pre-treatment. However the diminish of staining was not significant difference between IP and IT PBN pre-treated rats.

Conclusion: This study suggests that systemic ROS scavenger posses the antinociceptive property in rats subjected to the formalin test, and ROS are involved in the development and maintenance of pain.

Reference: J Pharmacol Exp Ther. 2004 Jun; 309(3): 869-78. 